\title{
Surface Plasmon Resonance-Based Fiber Optic Sensors: Principle, Probe Designs, and Some Applications
}

\author{
B. D. Gupta and R. K. Verma \\ Department of Physics, Indian Institute of Technology Delhi, New Delhi 110016, India \\ Correspondence should be addressed to B. D. Gupta, bdgupta@physics.iitd.ernet.in
}

Received 26 January 2009; Accepted 2 June 2009

Recommended by Christos Riziotis

\begin{abstract}
Surface plasmon resonance technique in collaboration with optical fiber technology has brought tremendous advancements in sensing of various physical, chemical, and biochemical parameters. In this review article, we present the principle of SPR technique for sensing and various designs of the fiber optic SPR probe reported for the enhancement of the sensitivity of the sensor. In addition, we present few examples of the surface plasmon resonance- (SPR-) based fiber optic sensors. The present review may provide researchers valuable information regarding fiber optic SPR sensors and encourage them to take this area for further research and development.
\end{abstract}

Copyright (C) 2009 B. D. Gupta and R. K. Verma. This is an open access article distributed under the Creative Commons Attribution License, which permits unrestricted use, distribution, and reproduction in any medium, provided the original work is properly cited.

\section{Introduction}

Surface plasmon resonance (SPR) is one of the most promising optical techniques that find applications in different fields. The first sensing application of SPR technique was reported in 1983 [1]. Since then, numerous SPR sensing structures for chemical and biochemical sensing have been reported. In SPR technique, a TM (transverse magnetic) or p-polarized light causes the excitation of electron density oscillations (known as surface plasmon wave, SPW) at the metal-dielectric interface. When the energy as well as the momentum of both, the incident light and SPW, match, a resonance occurs which results in a sharp dip in the reflected light intensity. The resonance condition depends on the angle of incidence, wavelength of the light beam, and the dielectric functions of both the metal as well as the dielectric. If the wavelength is kept constant and the angle of incidence is varied, then the sharp dip appears at a particular angle and the method is called angular interrogation. In another method, called spectral or wavelength interrogation, the angle of the incident beam is kept constant and the wavelength is varied. In this method, resonance occurs at a particular wavelength. The resonance parameter (angle or wavelength) depends on the refractive index of the dielectric medium. Change in refractive index changes the value of the resonance parameter. To excite surface plasmons, generally, a prism is used [2-9]. The prism-based SPR sensing device has a number of shortcomings such as its bulky size and the presence of various optical and mechanical (moving) parts. Further, the prism-based SPR sensing device cannot be used for remote sensing applications. These shortcomings can be overcome if an optical fiber is used in place of prism. The additional advantage of optical fiber is that the SPR probe can be miniaturized which can be advantageous for samples which are available in minute quantity or are costly. Due to these advantages the surface plasmon resonance-based optical fiber sensors have drawn a lot of attention [10-23]. Both experimental and theoretical investigations have been reported in literature on the SPRbased fiber optic sensors. The performance of these sensors is, generally, evaluated in terms of sensitivity and signalto-noise ratio (or detection accuracy). As is known, the higher the values of these parameters, the better is the sensor.

The present review begins with a section on principle of the sensing technique. The section includes the description of the performance parameters of the sensor: sensitivity and signal-to-noise ratio or detection accuracy. In the next section, we present the simplest SPR-based fiber optic sensor and discuss its modulation scheme. Section 4 of the present 
review deals with various designs of the SPR probe studied to enhance the performance of the fiber optic sensor. The designs include tapered, U-shaped and side-polished. The advantages of bimetallic coatings, addition of dopants in fiber core and the choice of the metals for coating have been discussed in the same section. In the last section, we review some of the SPR-based fiber optic sensors in details that have been reported in literature.

\section{Principle}

A metal-dielectric interface supports charge density oscillations along the interface which are called surface plasma oscillations. The quantum of these oscillations is given the name as surface plasmon. The surface plasmons are accompanied by a longitudinal (TM- or p-polarized) electric field which decays exponentially in metal as well as in dielectric medium. The electric field has its maximum at metaldielectric interface. The TM-polarization and exponential decay of electric field are found by solving the Maxwell equation for semi-infinite media of metal and dielectric with an interface of metal-dielectric. The propagation constant $\left(K_{\mathrm{SP}}\right)$ of the surface plasmon wave propagating along the metal-dielectric interface is given by

$$
K_{\mathrm{SP}}=\frac{\omega}{c}\left(\frac{\varepsilon_{m} \varepsilon_{s}}{\varepsilon_{m}+\varepsilon_{s}}\right)^{1 / 2},
$$

where $\varepsilon_{m}$ and $\varepsilon_{s}$ are the dielectric constants of metal and the dielectric medium, respectively, $\omega$ is the frequency of incident light, and $c$ is the velocity of light. From (1) it may be noted that the propagation constant of surface plasmon wave depends on the dielectric constants of both the metal and the dielectric medium.

The surface plasmons can be excited by light with same polarization state as that of surface plasmons. The propagation constant $\left(K_{s}\right)$ of the light wave with frequency $\omega$ propagating through the dielectric medium is given by

$$
K_{s}=\frac{\omega}{c} \sqrt{\varepsilon_{s}}
$$

Since $\varepsilon_{m}<0$ (for metal) and $\varepsilon_{s}>0$ (for dielectric), for a given frequency, the propagation constant of surface plasmon $\left(K_{\mathrm{SP}}\right)$ is greater than that of the light wave in dielectric medium $\left(K_{s}\right)$. To excite surface plasmons, two propagation wave-vectors should be equal. Hence, the direct light cannot excite surface plasmons at a metal-dielectric interface. To excite surface plasmons the momentum and hence the wave vector of the exciting light in dielectric medium should be increased. This can be done if instead of a direct light, evanescent wave is used to excite the surface plasmons. To obtain the evanescent wave for the excitation of surface plasmons, a prism with high dielectric constant is used.

When a light beam is incident through one of the two sides of the prism at an angle greater than the critical angle at prism-air interface the total internal reflection of light beam takes place. In the condition of total internal reflection light beam does not return exactly from the

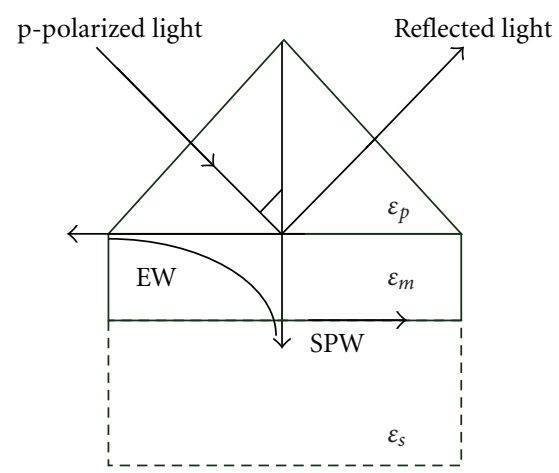

FIGURE 1: Kretschmann configuration for the excitation of surface plasmon at metal-dielectric interface [23]. (C) IEEE.

interface. Instead it returns after penetrating in the lower refractive index medium (air in this case). The field in the lower refractive index medium is called evanescent field and the wave corresponding to this is called evanescent wave. The evanescent wave propagates along the prism-air interface and decays exponentially in the rarer medium (air). The propagation constant of the evanescent wave at prism-air interface is given by

$$
K_{\mathrm{ev}}=\frac{\omega}{c} \sqrt{\varepsilon_{p}} \sin \theta,
$$

where $\varepsilon_{p}$ represents the dielectric constant of the material of the prism and $\theta$ is the angle of incidence of the beam. Increase in the dielectric constant of the prism increases the propagation constant of the evanescent wave and hence this can be made equal to propagation constant of the surface plasmon wave to satisfy the surface plasmon resonance condition. Kretschmann and Reather [24] devised a prismbased configuration, shown in Figure 1, to excite the surface plasmons using evanescent wave. In this configuration the base of the glass prism is coated with a thin layer of metal (typically around $50 \mathrm{~nm}$ ). The metal layer is kept in direct contact with the dielectric medium of lower refractive index (such as air or some other dielectric sample). When a p-polarized light beam is incident through the prism on the prism-metal layer interface at an angle $\theta$ equal to or greater than the critical angle, the evanescent wave is produced at the prism-metal interface. The excitation of surface plasmons occurs when the wave vector of evanescent wave exactly matches with that of the surface plasmons of similar frequency. This occurs at a particular angle of incidence $\theta_{\text {res }}$. Thus the resonance condition for surface plasmon resonance is

$$
\frac{\omega}{c} \sqrt{\varepsilon_{p}} \sin \theta_{\mathrm{res}}=\frac{\omega}{c}\left(\frac{\varepsilon_{m} \varepsilon_{s}}{\varepsilon_{m}+\varepsilon_{s}}\right)^{1 / 2}
$$

The excitation of surface plasmons at metal/dielectric interface results in the transfer of energy from incident light to surface plasmons, which reduces the intensity of the reflected light. If the intensity of the reflected light is measured as a function of angle of incidence $\theta$ for fixed values of frequency, metal layer thickness and dielectric layer thickness then a 


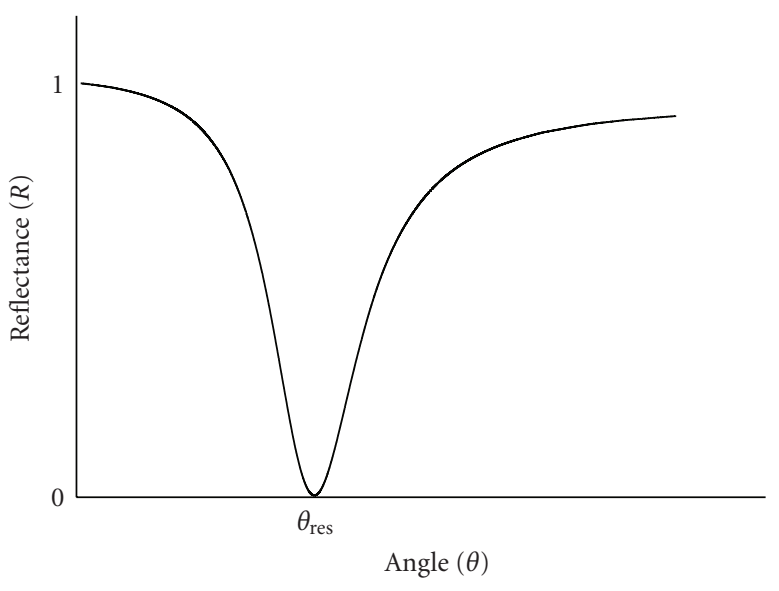

FIgURE 2: SPR spectrum [23]. (c) IEEE.

sharp dip is observed at resonance angle, $\theta_{\text {res }}$, due to an efficient transfer of energy to surface plasmons as shown in Figure 2. The minimum of the reflected intensity can be quantitatively described with the help of Fresnel's equations for the three-layer system.

For a given frequency of the light source and the dielectric constant of metal film one can determine the dielectric constant $\left(\varepsilon_{s}\right)$ of the sensing layer adjacent to metal layer using (4) if the value of the resonance angle $\left(\theta_{\text {res }}\right)$ is known. The resonance angle is experimentally determined by using angular interrogation method. It is very sensitive to variation in the refractive index of the sensing layer. Increase in refractive index of the sensing layer increases the resonance angle.

Sensitivity and detection accuracy or signal-to-noise ratio (SNR) are the two parameters that are used to analyze the performance of an SPR sensor. For the best performance both the parameters should be as high as possible. Sensitivity of an SPR sensor utilizing angular interrogation method depends on the amount of shift of the resonance angle with a change in the refractive index of the sensing layer. For a given refractive index change if the shift in resonance angle increases this means an increase in the sensitivity of the sensor.

Figure 3 shows a plot of reflectance as a function of angle of incidence of the light beam for sensing layer with refractive indices $n_{s}$ and $n_{s}+\delta n_{s}$. Increase in refractive index by $\delta n_{s}$ shifts the resonance angle by $\delta \theta_{\text {res }}$. Thus the sensitivity of an SPR sensor utilizing angular interrogation method is defined as

$$
S_{n}=\frac{\delta \theta_{\text {res }}}{\delta n_{s}}
$$

The detection accuracy or the SNR of an SPR sensor depends on how accurately and precisely the sensor can detect the resonance angle and hence, the refractive index of the sensing layer. The narrower the width of the SPR curve, the higher is the detection accuracy. Therefore, if $\delta \theta_{0.5}$ is the angular width of the SPR curve corresponding to reflectance 0.5 , the detection accuracy of the sensor is assumed to be

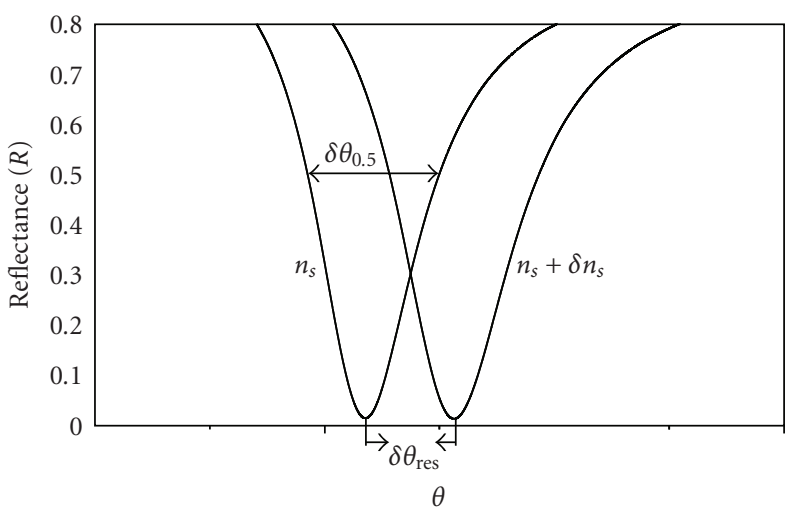

FIGURE 3: SPR spectra for two different refractive indices of the sensing layer [23]. (C) IEEE.

inversely proportional to $\delta \theta_{0.5}$ (Figure 3). The SNR of the SPR sensor with angular interrogation is, thus, defined as [25]

$$
\mathrm{SNR}=\frac{\delta \theta_{\text {res }}}{\delta \theta_{0.5}}
$$

\section{Fiber Optic SPR-Based Sensors}

In the case of a prism-based SPR sensor evanescent wave required to excite surface plasmons is resulted due to the total internal reflection taking place at the prism-metal interface when the angle of incidence of the beam is greater than the critical angle. The evanescent wave is also present in an optical fiber because the light guidance in an optical fiber occurs due to the total internal reflection of the guided ray at the core-cladding interface. In the case of optical fiber the evanescent wave propagates along the corecladding interface. Therefore, to design an SPR-based fiber optic sensor, the prism can be replaced by the core of an optical fiber. To fabricate an SPR-based fiber optic sensor, the silicon cladding from a small portion of the fiber, preferably from the middle, is removed and the unclad core is coated with a metal layer. The metal layer is further, surrounded by a dielectric sensing layer as shown in Figure 4. In an SPR-based fiber optic sensor, all the guided rays are launched and hence, instead of angular interrogation, spectral interrogation method is used. The light from a polychromatic source is launched into one of the ends of the optical fiber. The evanescent field produced by the guided rays excites the surface plasmons at the metal-dielectric sensing layer interface. The coupling of evanescent field with surface plasmons strongly depends on wavelength, fiber parameters, probe geometry, and the metal layer properties. Unlike prism-based SPR sensor, the number of reflections for most of the guided rays is greater than one in SPR-based fiber optic sensor geometry. The smaller the angle of incidence at the interface, the larger is the number of reflections per unit length in the fiber. In addition, the number of reflections for any ray also depends on the length of the sensing region 


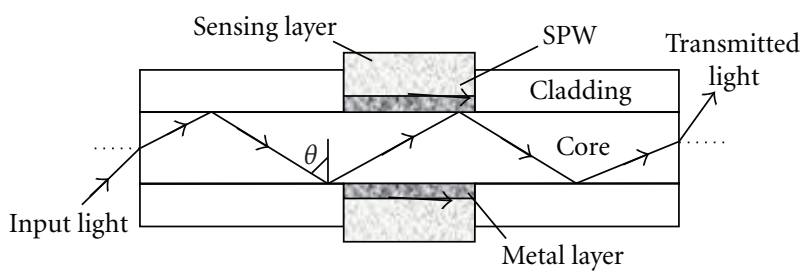

FIGURE 4: A typical probe of an SPR-based fiber optic sensor [23]. (C) IEEE.

and the fiber core diameter. The number of reflections is one of the important parameters that affect the width of the SPR curve. The intensity of the light transmitted after passing through the SPR sensing region is detected at the other end of the fiber as a function of wavelength. The SPR spectrum thus obtained is similar in shape to that shown in Figure 2. The sensing is accomplished by observing the wavelength corresponding to the dip in the spectrum (called resonance wavelength). A plot of resonance wavelength with the refractive index of the sensing layer is the calibration curve of the SPR sensor. The sensitivity and the detection accuracy are determined in the same way as determined in the case of angular interrogation. The angles are replaced by wavelengths in the definitions of sensitivity and detection accuracy.

\section{SPR Probe Designs}

Sensitivity, detection accuracy, reproducibility, and operating range of a sensor are the important parameters to compare it with other sensors. The best sensor is the one that has high sensitivity, detection accuracy and operating range, in addition to giving reproducible results. To achieve this various modifications have been carried out in the design of fiber optic SPR probe. We review some of these modifications in what follows.

4.1. Bimetallic Coating. For metallic coating on prism base or fiber core either silver or gold is used. Gold demonstrates a higher shift of resonance parameter to change in refractive index of sensing layer and is chemically stable. Silver, on the other hand, displays a narrower width of the SPR curve causing a higher SNR or detection accuracy. The sharpness of the resonance curve depends upon the imaginary part of the dielectric constant of the metal. Silver having the larger value of the imaginary part of the dielectric constant shows narrower width of the SPR curve causing a higher SNR or detection accuracy. On the other hand, the shift of the resonance curve depends on the real part of the dielectric constant of the metal. The real part of the dielectric constant is large in the case of gold than the silver and hence gold demonstrates a higher shift of resonance parameter to change in refractive index of sensing layer. The chemical stability of silver is poor due to its oxidation. The oxidation of silver occurs as soon as it is exposed to air and especially to water, which makes it difficult to give a reproducible result and hence the sensor remains unreliable for practical applications. Therefore, the treatment of silver surface by a thin and dense cover is required. In this regard, a new structure of resonant metal film based on bimetallic layers (gold as outer) on the prism base with angular interrogation method was reported [25]. The new structure displayed a large shift of resonance angle as gold film, and also showed narrower resonance curve as silver film along with the protection of silver film against oxidation. The same structure with spectral interrogation was extended to SPRbased optical fiber sensor for the selected and all guided rays configurations [19]. The sensitivity and SNR were evaluated numerically for different ratios of the thicknesses of silver and gold layers. Figures 5(a) and 5(b) show the variations of SNR and sensitivity with percentage of silver in bimetallic combination, respectively [19]. As expected the SNR increases with the increase in the silver thickness. The variation is almost the same for both kinds of launching but the values of SNR for all guided rays launching are about 1.5 times higher than those corresponding to selected rays launching. As far as sensitivity is concerned, it decreases as the silver layer thickness increases or gold layer thickness decreases. Its variation with silver thickness is almost same in the two cases but in terms of values, the selected ray launching has higher value than the all guided rays launching case.

4.2. Choice of Metals. The capability of other metals such as copper $(\mathrm{Cu})$ and aluminium $(\mathrm{Al})$ for SPR sensor applications has also been analyzed. Both metals have the ability to be used for an SPR sensor. The copper has some limitations like silver. It is chemically vulnerable against oxidation and corrosion, therefore, its protection is required for a stable sensing application. The SPR sensing capabilities of different bimetallic combinations made out of $\mathrm{Ag}, \mathrm{Au}, \mathrm{Al}$, and $\mathrm{Cu}$ were theoretically investigated for the design of SPR-based fiber optic sensors [26]. Figures 6(a) and 6(b) show the variation of sensitivity and SNR with the ratio of inner layer thickness to total bimetallic thickness for different bimetallic combinations, respectively [26]. The figure predicts that the sensor with single gold layer is the most sensitive whereas the sensor with single aluminium layer is the least. Further, $\mathrm{Cu}-\mathrm{Al}$ combination provides the minimum sensitivity for any ratio of their corresponding thickness values. In all the combinations with gold, $\mathrm{Ag}-\mathrm{Au}$ and $\mathrm{Cu}-$ $\mathrm{Au}$ combinations provide good sensitivity for the small thickness of the inner layer while the Au-Al combination provides larger sensitivity than all other combinations for the larger thickness of inner gold layer. It implies that a thick Au layer with very thin cover of Al layer (around 2$4 \mathrm{~nm}$ ) provides quite a large sensitivity. As far as variation of SNR with inner layer fraction is concerned, $\mathrm{Cu}-\mathrm{Al}$ is better among all the bimetallic combinations while the Ag$\mathrm{Au}$ combination provides the minimum values of SNR. To achieve highest SNR, one should choose a thin $\mathrm{Cu}$ layer and a much thicker covering of Al layer. This study implies that there is no single combination of metals that provides high values of both SNR and sensitivity simultaneously. 


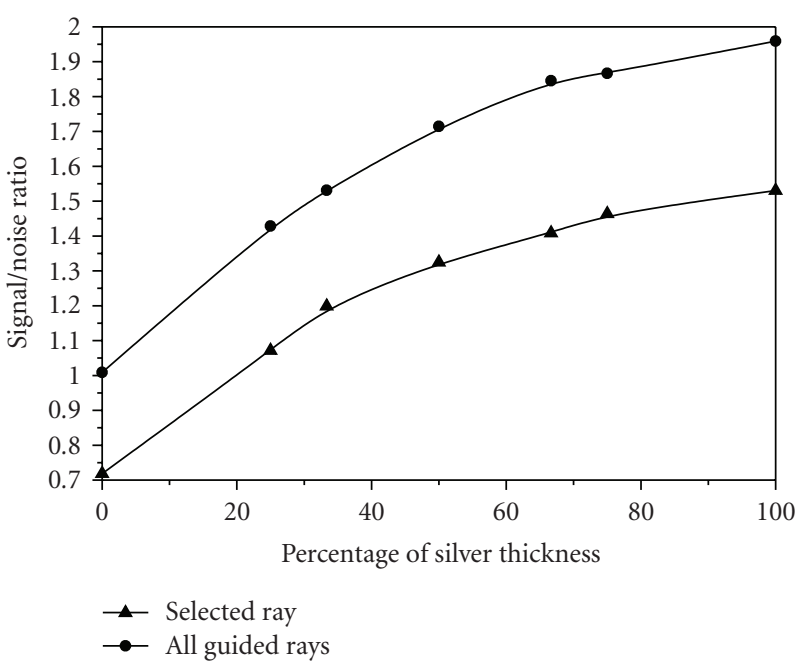

(a)

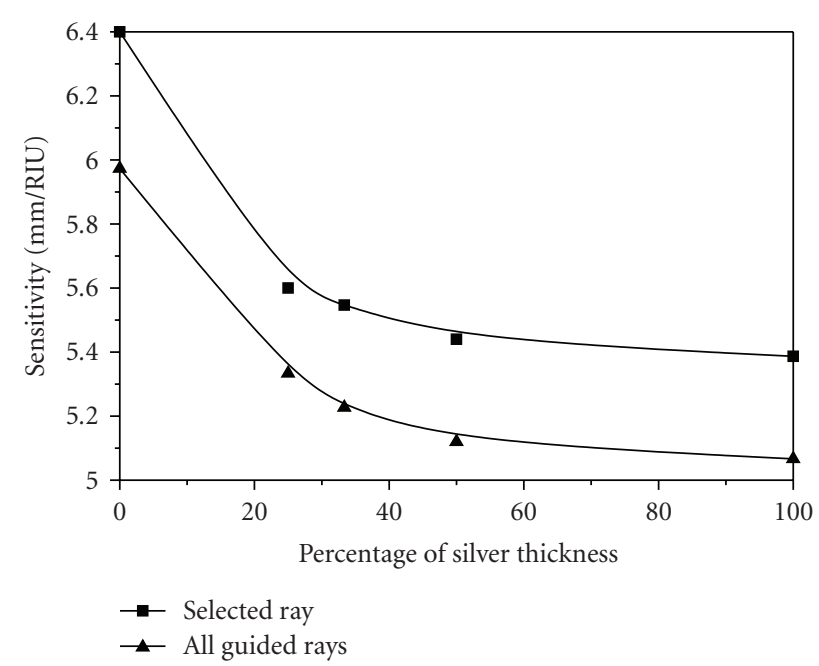

(b)

FIGURE 5: Variation of (a) signal-to-noise ratio and (b) sensitivity with percentage of silver in bimetallic layer for two different kinds of light launching [19]. (C) Elsevier.

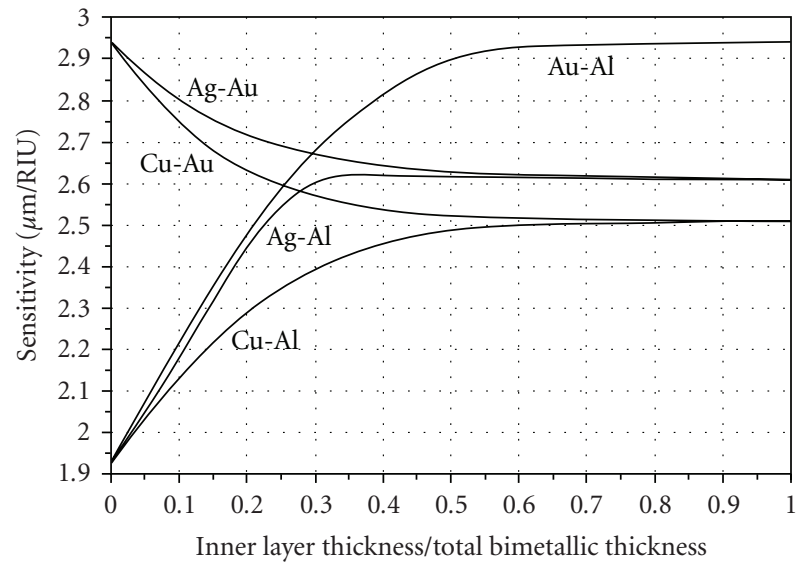

(a)

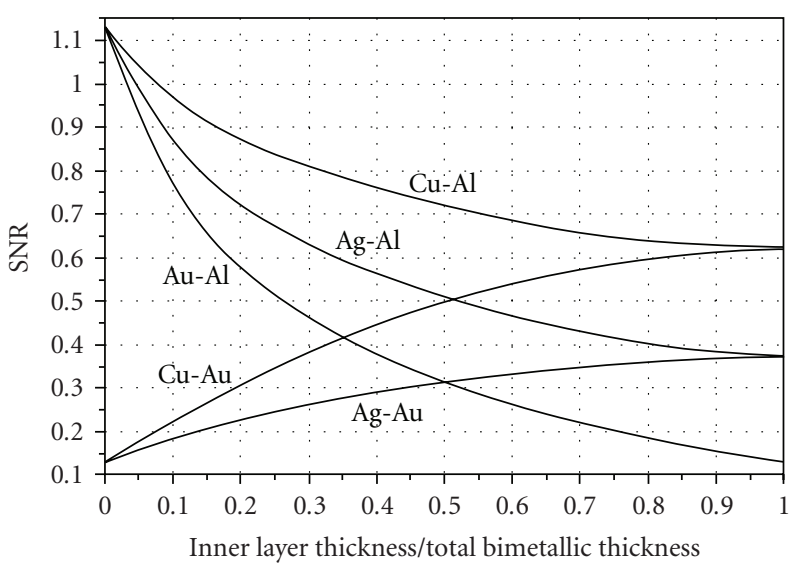

(b)

FIGURE 6: Variation of (a) sensitivity and (b) SNR with inner layer fraction for different bimetallic combinations [26]. (c) AIP.

4.3. Effect of Dopants. It may be noted from (4) that the SPR condition depends upon the refractive index of the material of the fiber core. Therefore, if an optical fiber is fabricated by adding dopants in the fiber core the sensitivity of the sensor can be enhanced or tuned. Generally, an optical fiber with pure silica core is used for SPR-based sensors. Sharma et al. [27] carried out theoretical modeling and analysis of SPRbased fiber optic sensor to evaluate the effect of dopants on the sensitivity and SNR. Germanium oxide $\left(\mathrm{GeO}_{2}\right)$, boron oxide $\left(\mathrm{B}_{2} \mathrm{O}_{3}\right)$, and phosphorus pent-oxide $\left(\mathrm{P}_{2} \mathrm{O}_{5}\right)$ were used as dopants for pure silica. Figure 7 shows the variation of sensitivity with the refractive index of the sensing layer for different dopants with concentrations [27]. The simulation predicts an increase of about 50\% in sensitivity between $\mathrm{B}_{2} \mathrm{O}_{3}$ (5.2) and $\mathrm{GeO}_{2}$ (19.3) dopants. Moreover, as the doping concentration of $\mathrm{GeO}_{2}$ is increased from a low of 6.3 mole $\%$ to a high of 19.3 mole $\%$, a noticeable decrease in sensor's sensitivity is obtained. Further, the effect of dopants on the sensitivity of the fiber optic SPR sensor was reported to be same irrespective of whether a single metal layer or a bimetallic configuration is used.

4.4. Tapered Probe. Several groups have worked on the improvement of the sensitivity of a fiber optic SPR sensor by changing the shape or the geometry of the fiber optic SPR probe. Tapering the fiber optic SPR probe was one of the modifications reported in literature [18, 28]. A typical tapered fiber optic SPR probe is shown in Figure 8 [29]. The uses of dual-tapered and tetra-tapered fiber optic SPR probes for gas and liquid sensing have also been reported [18]. Changing the profile of the tapered SPR probe also affects the sensitivity of the sensor. Surface plasmon resonance-based tapered fiber optic sensor with three different taper profiles, 


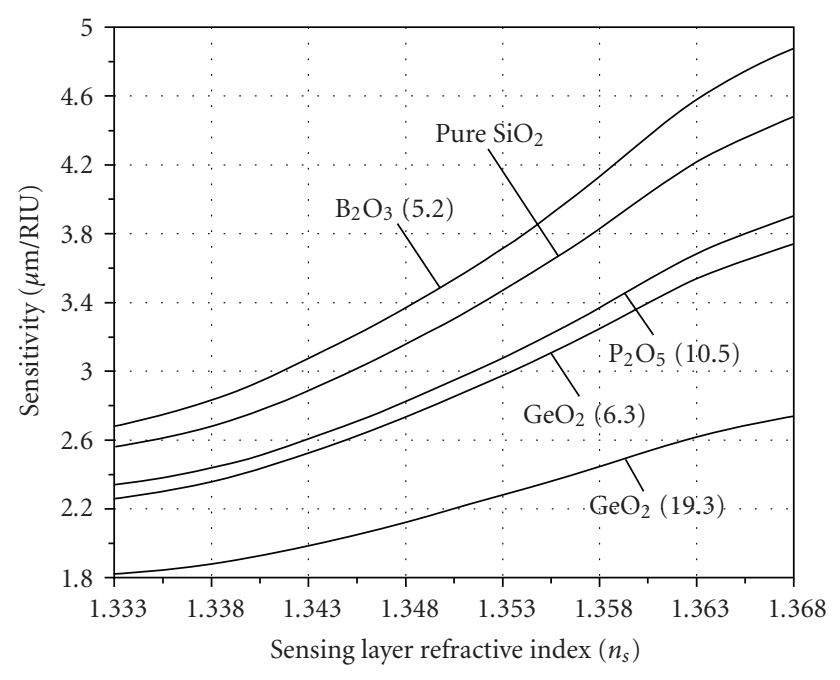

FIGURE 7: Variation of sensitivity with sensing layer refractive index. A: Silica doped with $\mathrm{GeO}_{2}$ (19.3); B: Silica doped with $\mathrm{GeO}_{2}$ (6.3); C: Silica doped with $\mathrm{P}_{2} \mathrm{O}_{5}$ (10.5); D: Pure silica with no doping; and E: Silica doped with $\mathrm{B}_{2} \mathrm{O}_{3}$ (5.2). Numbers in brackets are the molar concentrations of dopant in mole \% [27]. (c) Elsevier.

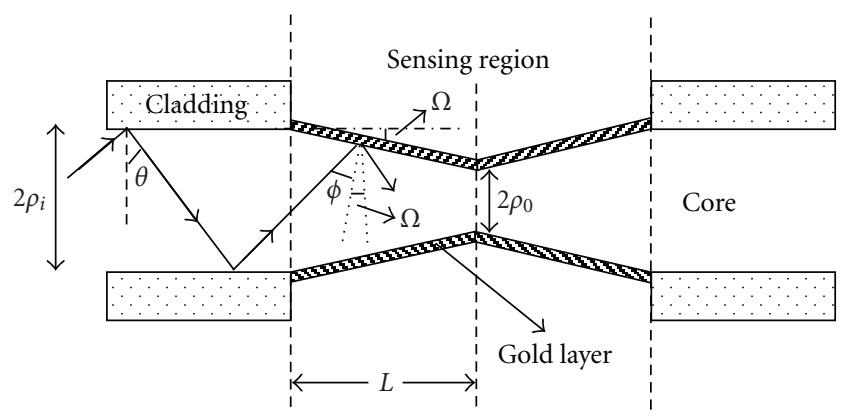

FIGURE 8: A typical SPR-based fiber optic sensor with tapered probe [29]. (C) Elsevier.

namely, linear, parabolic, and exponential-linear, shown in Figure 9, was analyzed theoretically [29]. Figure 10 shows the variation of the sensitivity of the tapered fiber optic SPR probe with taper ratio for these three taper profiles [29]. Theoretical analysis predicts an increase in the sensitivity with the increase in the taper ratio. The study further shows that, for a given taper ratio, the exponential-linear taper profile provides the maximum sensitivity. The increase in sensitivity occurs because of the decrease in the angle of incidence of the guided rays with the normal to the corecladding interface in the tapered region.

To further enhance the sensitivity, an SPR probe of uniform core (with metallic coating) sandwiched between two unclad tapered fiber regions, shown in Figure 11, was proposed [30]. The taper region 1 brings down the angles of the bound rays in the fiber close to the critical angle of the unclad tapered region while the taper region 2 reconverts the angles of these rays to their initial values so that all the guided rays can propagate up to the output end of the fiber. This was achieved by choosing the minimum allowed value

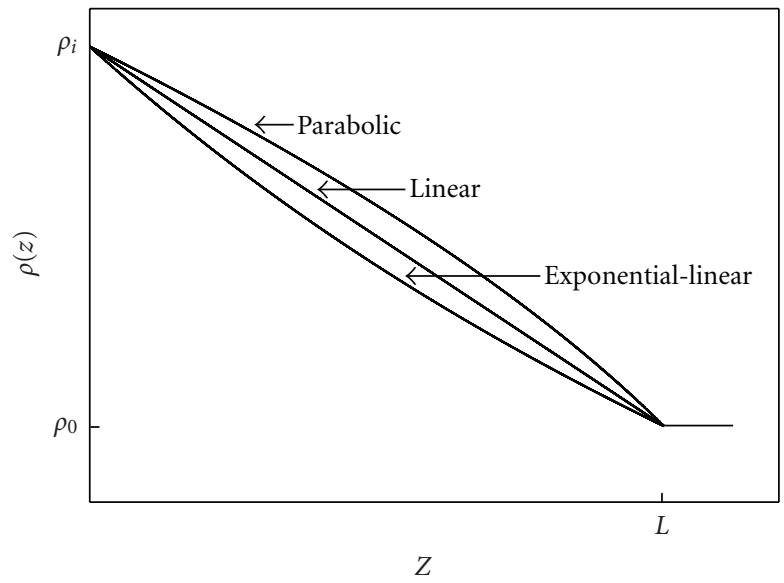

Figure 9: Three different taper profiles showing the linear, parabolic, and exponential-linear [29]. (C) Elsevier.

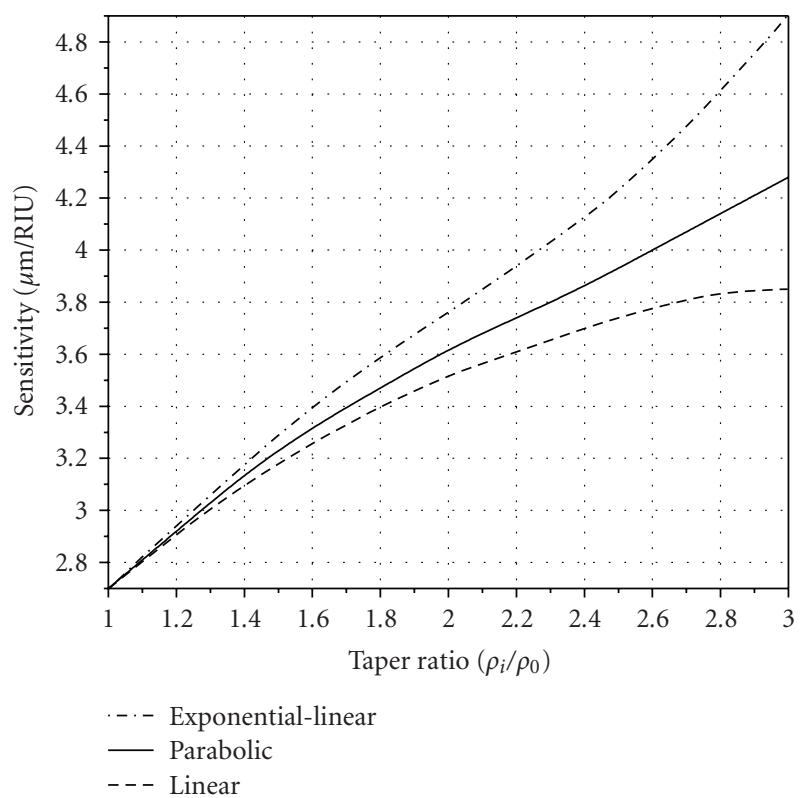

FIGURE 10: Variation of sensitivity with taper ratio for three different taper profiles [29]. (c) Elsevier.

of the radius of the uniform core in the sensing region. In the sensing region rays propagate close to the critical angle of the region. Figure 12 shows the variation of sensitivity with taper ratio for this kind of probe [30]. The sensitivity increases with an increase in the taper ratio as reported in other studies [29]. However, a significant sensitivity enhancement of more than 5 times was obtained for a taper ratio of 2.0 in comparison to conventional $(\mathrm{TR}=1)$ fiber optic SPR sensor.

4.5. U-Shaped. The angle of incidence of the ray with the normal to the core-cladding interface can be brought close to the critical angle by using a U-shaped probe. An SPRbased fiber optic sensor with uniform semimetal coated Ushaped probe, shown in Figure 13, was analyzed using a bidimensional model [31]. To make the analysis simpler, all 


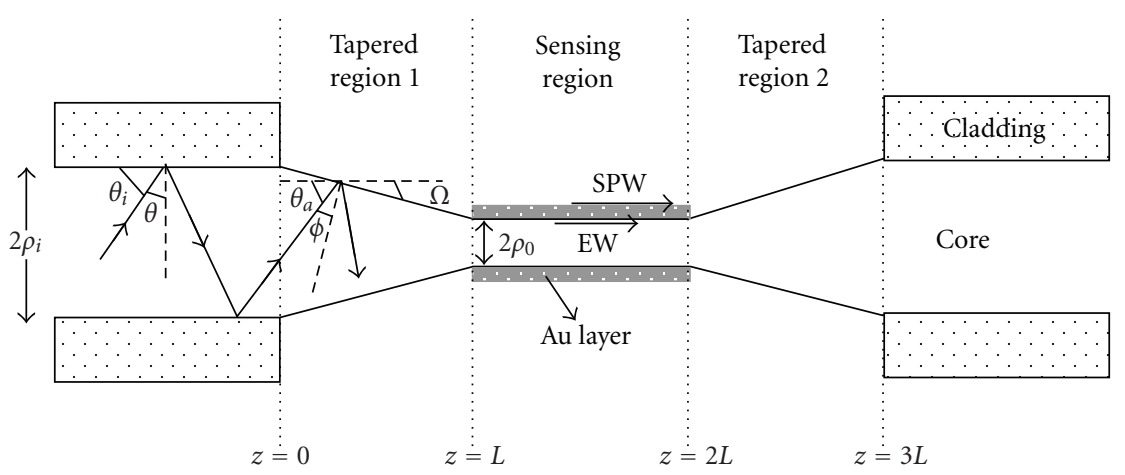

FIGURE 11: A novel SPR-based fiber optic sensor. Sensing probe of uniform core radius is sandwiched between two tapered fiber regions [30]. (C) IEEE.

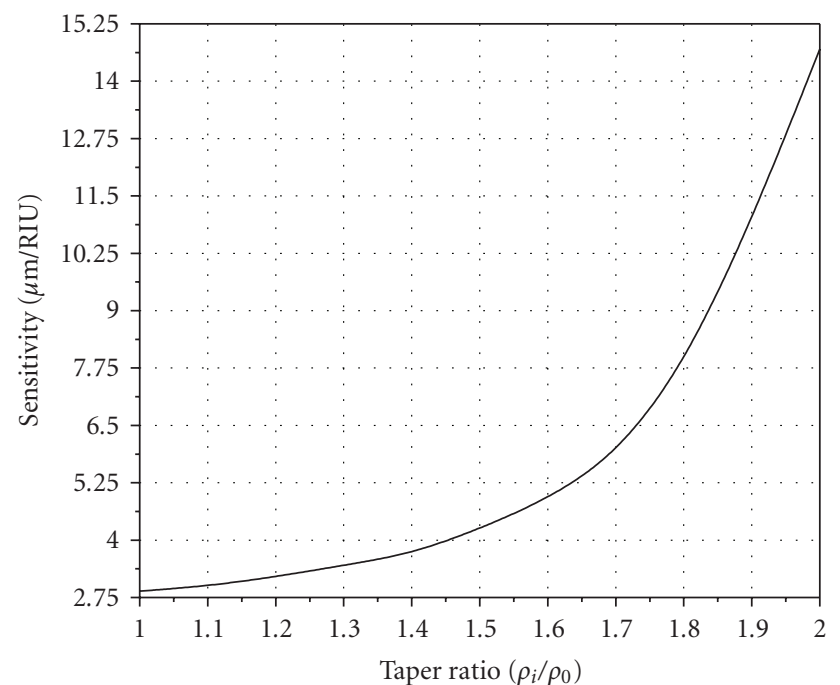

FIGURE 12: Variation of sensitivity of the SPR-based fiber optic sensor with taper ratio [30]. (C) IEEE.

the guided rays of the p-polarized light launched in the fiber and their electric vectors were assumed to lie in the plane of bending of the U-shaped probe. Figure 14 shows the variation of the sensitivity of the probe with bending radius for different values of the sensing length of the probe. Increase in sensitivity with the decrease in the bending radius was obtained. The increase in sensitivity was up to a certain value of the bending radius below that it starts decreasing sharply. The decrease in sensitivity occurs because the angle of incidence of the last ray becomes less than the critical angle required for a ray to be guided in the bent region. Thus, there exists an optimum value of the bending radius at which the sensitivity of the SPR sensor based on U-shaped probe acquires a maximum value. The trend of variation was same for all the three values of the sensing length used. This is due to the independence of the angle of incidence on the sensing length. For a given bending radius the sensitivity increases as the sensing length increases. The increase in sensitivity, as mentioned earlier, is due to the decrease in the angle of incidence. The decrease in angle of incidence increases the

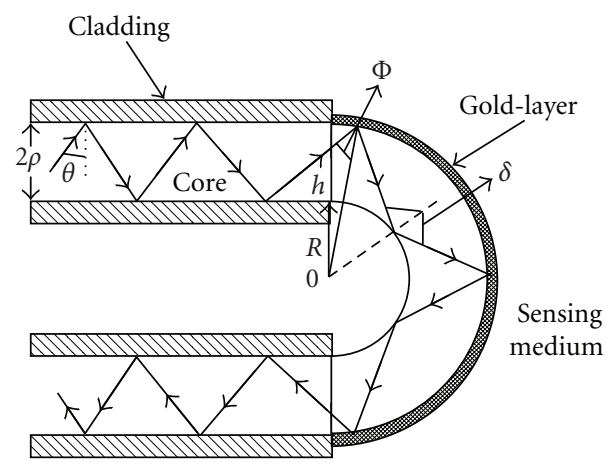

FIGURE 13: A typical U-shaped fiber optic SPR probe [31]. (c) IOP.

number of reflections which causes the broadening of SPR curve and hence the decrease in the detection accuracy or SNR of the sensor. The enhancement in sensitivity obtained was much more compared to the decrease in the detection accuracy and hence the decrease in detection accuracy can be tolerated. In fact the maximum sensitivity achieved was several times more than that reported for an SPR-based fiber optic tapered probe.

4.6. Side-Polished Fiber. The SPR probes reported above use multimode optical fibers. A single mode fiber has also been used to fabricate fiber optic SPR probe. Surface plasmon resonance sensor using side-polished single mode optical fiber and a thin metal over layer is shown in Figure 15 [32]. The design of the probe is slightly different from that shown in Figure 4. In this configuration, the guided mode propagating in the fiber excites the surface plasmon wave at the interface between the metal and a sensing medium. The resonance occurs if the two modes are closely phase matched. Such single-mode optical fiber based SPR sensor is more sensitive and more accurate in comparison to those with multi-mode optical fibers. However, their fabrication is much more complex and sophisticated compared with those that use multi-mode fibers. The advantage of side-polished half block SPR sensor is that it requires a very small amount of sample for measuring the refractive index. 


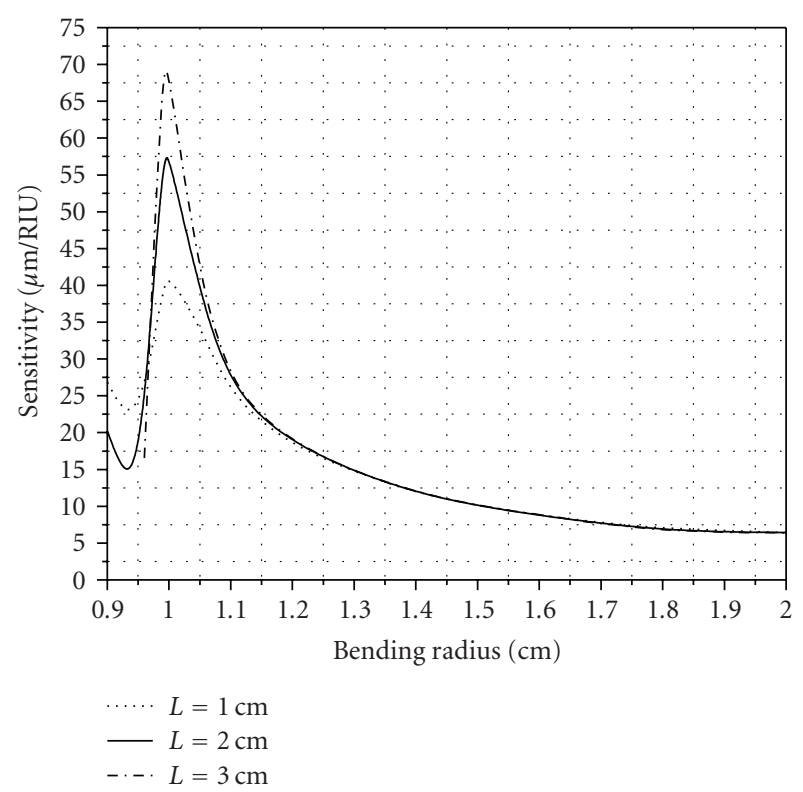

FIGURE 14: Variation of sensitivity with bending radius for three different values of the sensing length [31]. (C) IOP.

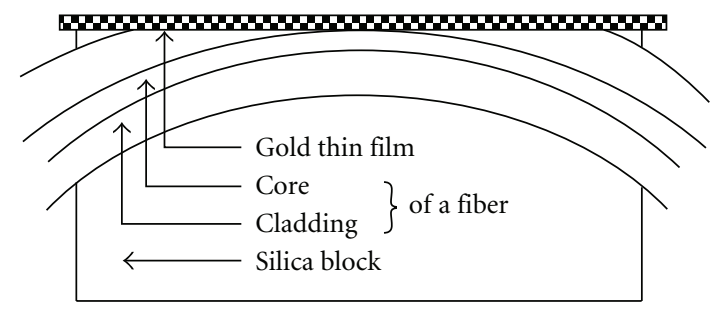

FIGURE 15: Side-polished single mode fiber optic SPR probe [23]. (C) IEEE.

Recently SPR-based side-polished multimode optical fiber sensors have been reported $[33,34]$. In these sensors the fiber was side-polished until half the core was closed. This increased the sensing area which is an advantage. Apart from side-polished single mode fiber, D-type single mode optical fibers have also been used for sensing applications utilizing SPR technique $[35,36]$. These fibers also improve sensitivity. The other designs for SPR-based fiber optic sensor includes SPR probe at one of the ends of the fiber with the reflecting end face $[37,38]$ and a fiber tip $[39,40]$. The photonic bandgap fiber based SPR sensors have also been reported very recently $[41,42]$.

4.7. Effect of Skew Rays. Most of the theoretical studies on fiber optic SPR sensors using ray optics and reported in literature do not consider skew rays in the analysis. These studies consider the propagation of only meridional rays in the fiber that makes the analysis simpler. However, apart from meridional rays, skew rays also exist in the fiber depending on the light launching conditions. These rays follow a helical path inside the fiber. To specify the trajectory of a skew ray, a second angle (known as skewness angle), in addition to the inclination of the ray with the axial direction of the fiber, is required. Recently, the effect of skew rays on the sensitivity and the SNR of a fiber optic SPR sensor were studied using spectral interrogation method [43]. Figures 16(a) and 16(b) show the variation of sensitivity and SNR with skewness parameter, respectively [43]. Both the sensitivity and the SNR decrease as the value of skewness parameter increases irrespective of the metal used for coating. The sensitivity is better in the case of gold whereas silver demonstrates better SNR as expected. The decrease in sensitivity for highest value of skewness parameter is about $6 \%$ in the case of both the metals. The effect of skew rays is more on SNR than on the sensitivity. In the case of gold film it is about $40 \%$ while in the case of silver it is around $30 \%$.

\section{Some Applications}

The surface plasmon resonance-based fiber optic sensors have large number of applications for quantitative detection of chemical and biological species. These include food quality, medical diagnostics and environmental monitoring. These are detected by changing the refractive index of the medium around the metallic coating. The measurand changes the refractive index of the medium directly or indirectly. Here we present few SPR-based fiber optic sensors.

5.1. Temperature. Since the refractive index of a medium depends on its temperature, the SPR technique can be applied to sense the temperature of a medium. The temperature sensor based on surface plasmon resonance was proposed using a coupling prism and angular interrogation mode of operation [44]. It was suggested that for SPR technique to be used in temperature sensing, the sensing layer of large thermo-optic coefficient (such as titanium dioxide or silicon acrylate) should be used. Further, the penetration of surface plasmon wave should be restricted only to metal and sensing layers by taking an appropriate thickness of the two layers. The analysis was later extended to SPR-based fiber optic remote sensor for temperature detection [45]. The sensing layer was assumed to be of $\mathrm{TiO}_{2}$ (titanium dioxide). The outermost ambient medium was chosen as air, which adds to the flexibility of the sensor's design. Figure 17 shows the variation in resonance wavelength with temperature for silver and gold [45]. For both the metals, resonance wavelength shifts to shorter side with the increase in the temperature. However, there is no appreciable difference in resonance wavelength (and hence, in temperature sensitivity) for two metals used. This was suggested to be due to the similarity in the variation of physical properties of both the metals with temperature. Figure 18 depicts the corresponding variation in SPR curve width with temperature [45]. The SPR curve width increases with the increase in the temperature. This was reported to happen because the imaginary (absorption) part of the metal dielectric function increases with temperature. The effect of other parameters such as numerical aperture and the ratio of sensing region length to fiber core diameter on the sensitivity and detection accuracy were also reported. 


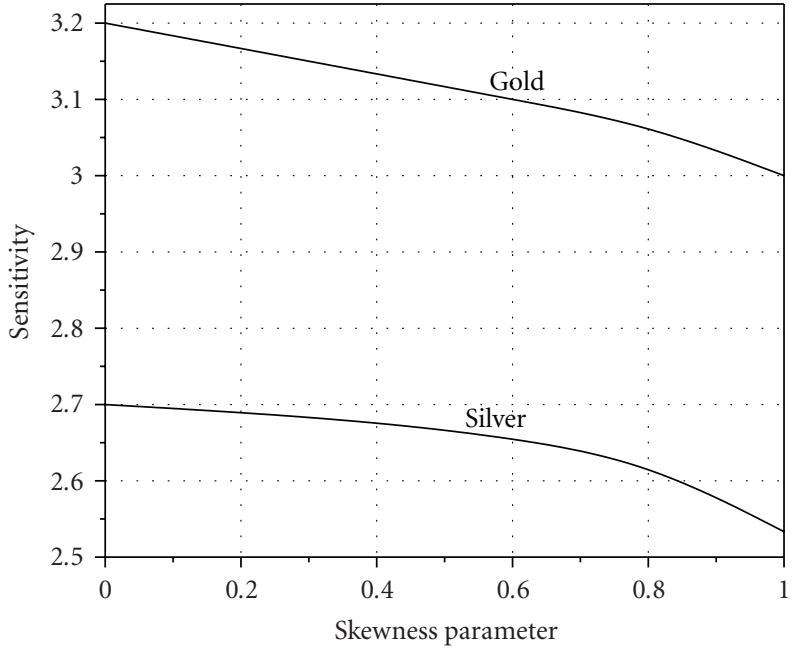

(a)

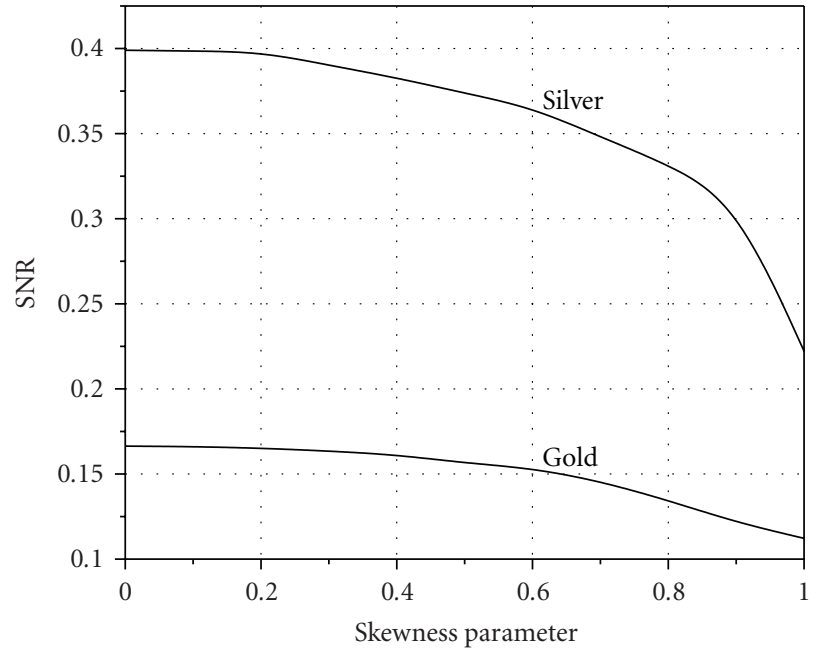

(b)

FIGURE 16: Variation of (a) Sensitivity and (b) SNR of an SPR-based fiber optic sensor with skewness parameter for two different metals [43]. (C) OSA.

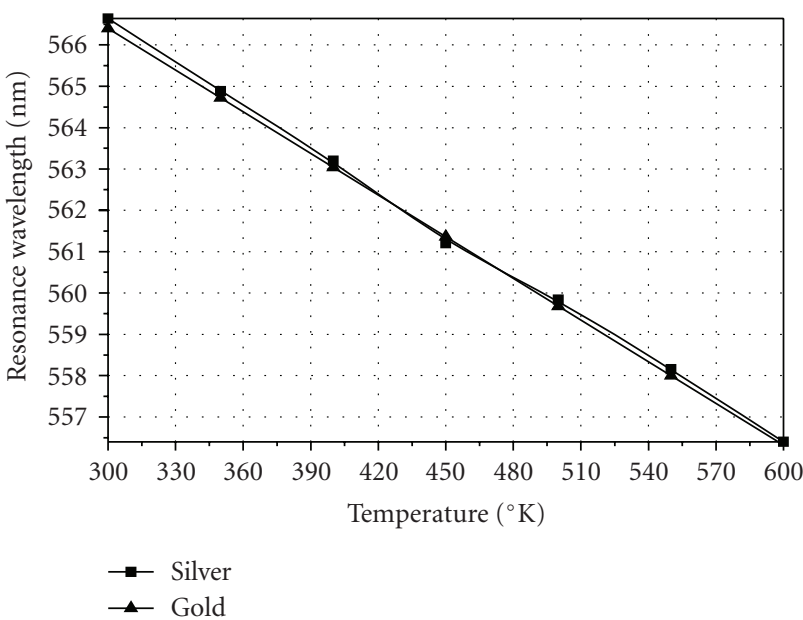

FIGURE 17: Variation of resonance wavelength with temperature for two metals: silver and gold [45]. (c) Elsevier.

It was also reported that the fiber with smaller numerical aperture provides larger detection accuracy without affecting the temperature sensitivity. Similarly, the small values of the ratio of sensing region length to fiber core diameter give high detection accuracy. For SPR-based fiber optic temperature sensor smaller sensing region and highly multimode optical fiber was recommended.

5.2. Naringin. The processing of citrus fruit juice has faced formidable problems in terms of bitterness, thereby affecting its consumer acceptability. The bitterness is caused by excessive naringin contents in fruit juice. The presence of naringin in fruit juice can be detected using an SPR-based fiber optic sensor. An SPR-based fiber optic sensor relying on spectral interrogation method was reported for the detection of

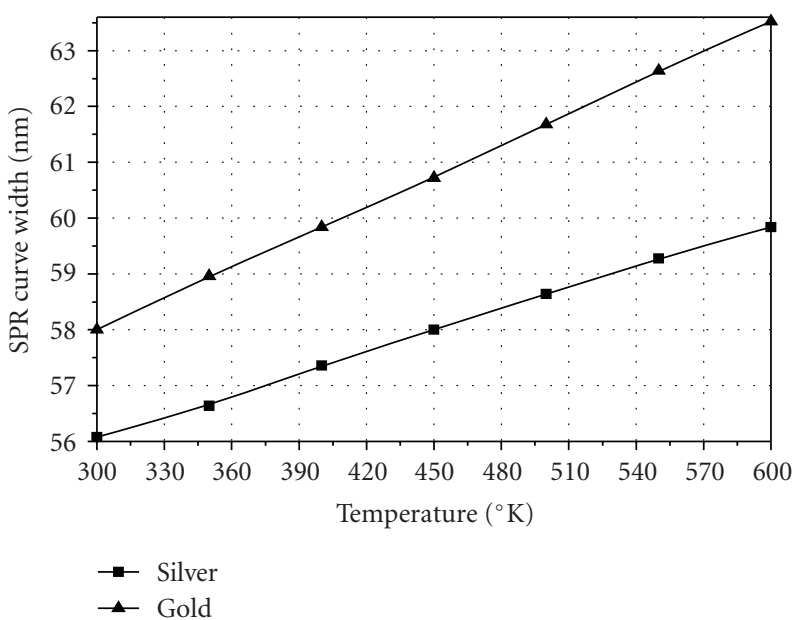

FIGURE 18: Variation of SPR curve width with temperature for two metals: silver and gold [45]. (c) Elsevier.

naringin [21]. The SPR probe was prepared by immobilizing the enzyme naringinase on the silver-coated core of the optical fiber. To immobilize, gel entrapment technique was used. The experimental set up for the characterization of the SPR-based fiber optic sensor is shown in Figure 19 [21]. Light from a broadband source (tungsten halogen lamp) was focused on the input face of the fiber using a circular slit and a microscope objective. The probe was mounted in a flow cell to keep the sample around the probe. The spectral distribution of the transmitted power for the sample around the probe was determined using a monochromator, a silicon detector and a power meter. The spectral distribution of transmitted light so obtained for a sample was divided by the corresponding spectral distribution obtained without any sample around the probe. The resultant SPR spectrum so obtained does not depend on the source spectral 


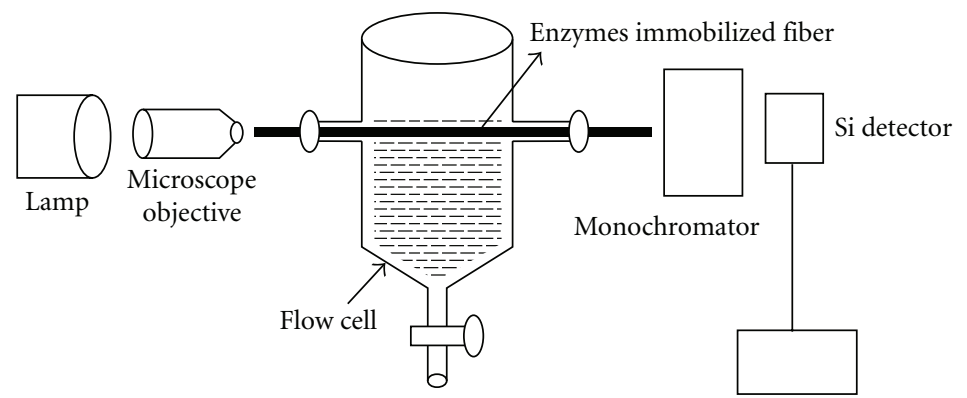

FIGURE 19: Experimental set up of the SPR-based fiber optic sensor [21]. (C) Elsevier.

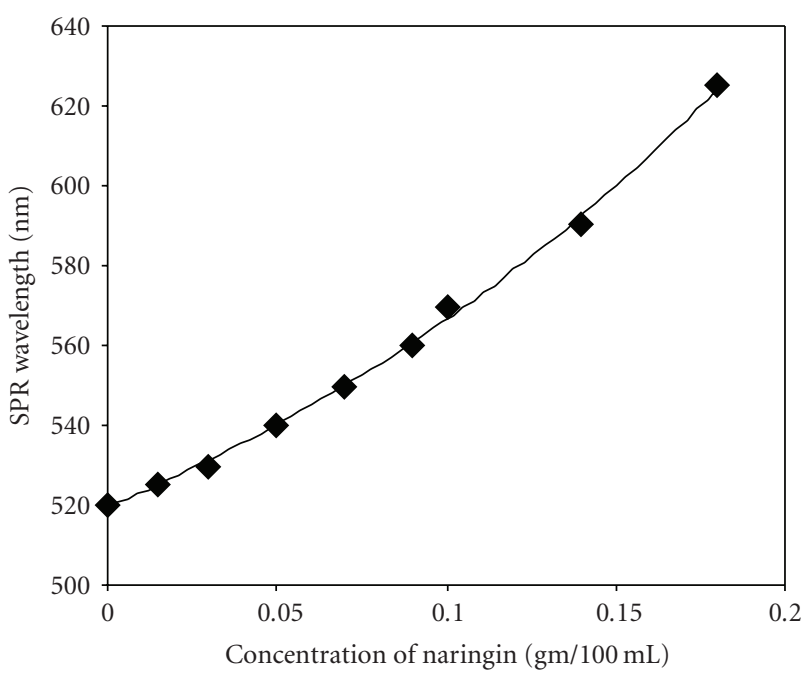

Figure 20: Variation of resonance wavelength with the concentration of naringin in buffer solution [21]. (c) Elsevier.

output, spectral sensitivity of photodetector and the spectral absorbance of the fiber. From the SPR spectrum resonance wavelength was determined. The calibration curve of the SPR-based fiber optic sensor obtained for the detection of naringin is shown in Figure 20 [21]. As the concentration of naringin increases the resonance wavelength increases. The increase in resonance wavelength was reported to be due to an increase in the refractive index of the immobilized layer which may occur due to the formation of a complex of naringin with the enzyme naringinase in the film.

5.3. Pesticides. Surface plasmon resonance-based fiber optic sensor for the detection of organophosphate pesticide, chlorphyrifos, was also reported recently using similar experimental method as used for the detection of naringin [46]. The probe was prepared by immobilizing acetylcholinesterase enzyme on the silver-coated core of the fiber. The principle of detection of pesticide was slightly different from that of naringin. It was based on the principle of competitive binding of the pesticide (acting as an inhibitor) for the substrate (acetlythiocholine iodide) to the enzyme. Figure 21 shows SPR spectra for pesticide concentration of $1.0 \mu \mathrm{M}$ and a substrate concentration of $2.5 \mathrm{mM}$ for the two cases

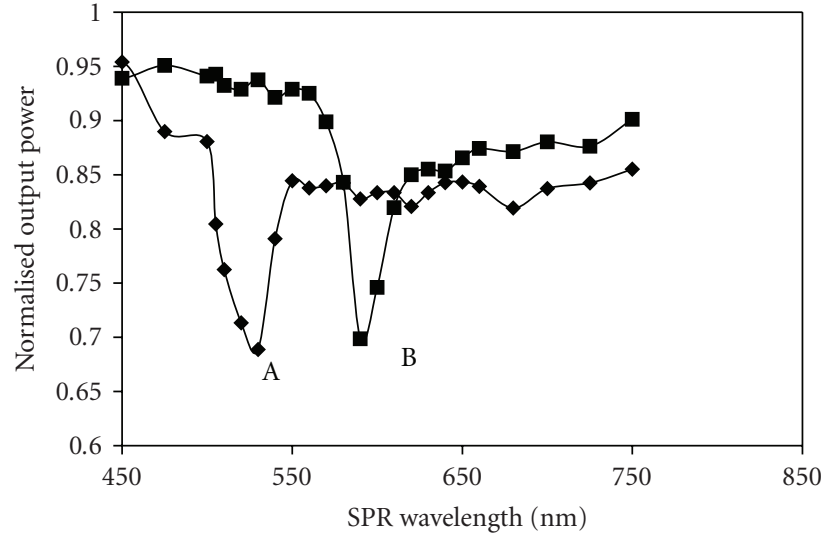

FIGURE 21: Variation of normalized output power as a function of wavelength for a substrate concentration $(2.5 \mathrm{mM})$ and $1.0 \mu \mathrm{M}$ concentration of pesticide (A) without enzyme and (B) with enzyme (B) [46]. (C) Elsevier.

(A) without enzyme (control experiment) and (B) with enzyme in the film [46]. The dip in control experiment occurs due to the refractive index of the gel. The difference in two spectra implies that the enzyme is playing a role in changing the refractive index of the gel in the presence of pesticide. The resonance wavelength was obtained around $590 \mathrm{~nm}$ for $1.0 \mu \mathrm{M}$ pesticide concentration. Figure 22 shows the calibration curve of the sensor [46]. The trend is opposite to that reported for the detection of naringin. In this case, the SPR wavelength decreases with the increase in the concentration of the pesticide for the fixed concentration of the substrate. The results appear to be due to the decrease in the refractive index of the film as the concentration of pesticide increases.

Surface plasmon resonance-based fiber optic sensors have also been reported for other chemical species. These include measurements of salinity in water [22, 37], refractive indices of alcohols [17], BSA [34], vapor and liquid analyses [18]. In addition a single-mode waveguide surface plasmon resonance sensor has been developed for biomolecular interaction analysis [47]. Recently, fiber optic SPR sensors have been reported that uses either a series of long period grating [48] or Bragg grating [49]. The advantage of these designs is that these offer multiple sensing channels capability. 


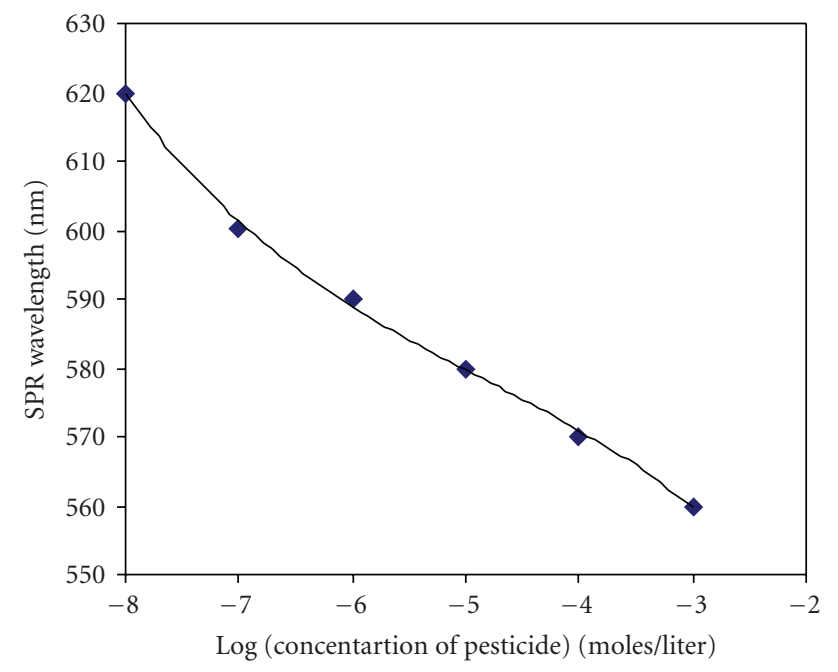

FIGURE 22: Calibration curve of SPR-based fiber optic pesticide sensor [46]. (C) Elsevier.

\section{Summary}

In this review article we have first described the principle of surface plasmon resonance technique. The technique is well established and will remain unchanged for years. In the beginning, the technique was used for prism-based SPR sensors but later when the advantages of optical fiber were realized, it was applied to optical fiber based sensors. Recently there have been significant advancements in both the design of fiber optic SPR sensors and strategies for the enhancement of the performance of the sensors particularly sensitivity. The probe designs include tapered, U-shaped, and side-polished fibers. These developments are likely to drive future trends in the research and development of optical fiber sensors. The unique optical properties of metal nanoparticles have also attracted the sensor community to develop localized surface plasmon resonance- (LSPR-) based sensors. The LSPR-based sensors have few advantages over bulk SPR-based sensors. However, the pace of collaboration of LSPR with optical fiber for sensing is at present slow but may gain momentum in coming years.

\section{Acknowledgment}

The present work is partially supported by the Department of Science and Technology (India).

\section{References}

[1] B. Liedberg, C. Nylander, and I. Lunström, "Surface plasmon resonance for gas detection and biosensing," Sensors and Actuators, vol. 4, pp. 299-304, 1983.

[2] J. van Gent, P. V. Lambeck, H. J. M. Kreuwel, et al., "Optimization of a chemooptical surface plasmon resonance based sensor," Applied Optics, vol. 29, pp. 2843-2849, 1990.

[3] E. Stenberg, B. Persson, H. Roos, and C. Urbaniczky, "Quantitative determination of surface concentration of protein with surface plasmon resonance using radiolabeled proteins,"
Journal of Colloid and Interface Science, vol. 143, no. 2, pp. 513526, 1991.

[4] G. Dougherty, "A compact optoelectronic instrument with a disposable sensor based on surface plasmon resonance," Measurement Science and Technology, vol. 4, no. 6, pp. 697699, 1993.

[5] S. Ekgasit, A. Tangcharoenbumrungsuk, F. Yu, A. Baba, and W. Knoll, "Resonance shifts in SPR curves of nonabsorbing, weakly absorbing, and strongly absorbing dielectrics," Sensors and Actuators B, vol. 105, no. 2, pp. 532-541, 2005.

[6] J.-J. Chyou, C.-S. Chu, F.-C. Chien, et al., "Precise determination of the dielectric constant and thickness of a nanolayer by use of surface plasmon resonance sensing and multiexperiment linear data analysis," Applied Optics, vol. 45, no. 23, pp. 6038-6044, 2006.

[7] H. P. Chiang, C.-W. Chen, J. J. Wu, et al., "Effects of temperature on the surface plasmon resonance at a metalsemiconductor interface," Thin Solid Films, vol. 515, no. 17, pp. 6953-6961, 2007.

[8] J. Le Person, F. Colas, C. Compere, et al., "Surface plasmon resonance in chalcogenide glass-based optical system," Sensors and Actuators B, vol. 130, no. 2, pp. 771-776, 2008.

[9] W. Feng, L. Shenye, P. Xiaoshi, C. Zhuangqi, and D. Yongkun, "Reflective-type configuration for monitoring the photobleaching procedure based on surface plasmon resonance," Journal of Optics A, vol. 10, no. 9, Article ID 095102, 2008.

[10] R. C. Jorgenson and S. S. Yee, "A fiber-optic chemical sensor based on surface plasmon resonance," Sensors and Actuators B, vol. 12, no. 3, pp. 213-220, 1993.

[11] R. D. Harris and J. S. Wilkinson, "Waveguide surface plasmon resonance sensors," Sensors and Actuators B, vol. 29, no. 1-3, pp. 261-267, 1995.

[12] W. B. Lin, N. Jaffrezic-Renault, A. Gagnaire, and H. Gagnaire, "The effects of polarization of the incident light-modeling and analysis of a SPR multimode optical fiber sensor," Sensors and Actuators A, vol. 84, no. 3, pp. 198-204, 2000.

[13] R. Slavík, J. Homola, J. Ctyroký, and E. Brynda, "Novel spectral fiber optic sensor based on surface plasmon resonance," Sensors and Actuators B, vol. 74, no. 1-3, pp. 106-111, 2001.

[14] M. Piliarik, J. Homola, Z. Maníková, and J. Ctyroký, "Surface plasmon resonance sensor based on a single-mode polarization-maintaining optical fiber," Sensors and Actuators $B$, vol. 90, no. 1-3, pp. 236-242, 2003.

[15] D. J. Gentleman, L. A. Obando, J.-F. Masson, J. R. Holloway, and K. S. Booksh, "Calibration of fiber optic based surface plasmon resonance sensors in aqueous systems," Analytica Chimica Acta, vol. 515, no. 2, pp. 291-302, 2004.

[16] A. K. Sharma and B. D. Gupta, "Absorption-based fiber optic surface plasmon resonance sensor: a theoretical evaluation," Sensors and Actuators B, vol. 100, no. 3, pp. 423-431, 2004.

[17] M. Mitsushio, S. Higashi, and M. Higo, "Construction and evaluation of a gold-deposited optical fiber sensor system for measurements of refractive indices of alcohols," Sensors and Actuators A, vol. 111, no. 2-3, pp. 252-259, 2004.

[18] Y.-C. Kim, W. Peng, S. Banerji, and K. S. Booksh, "Tapered fiber optic surface plasmon resonance sensor for analyses of vapor and liquid phases," Optics Letters, vol. 30, no. 17, pp. 2218-2220, 2005.

[19] A. K. Sharma and B. D. Gupta, "On the sensitivity and signal to noise ratio of a step-index fiber optic surface plasmon resonance sensor with bimetallic layers," Optics Communications, vol. 245, no. 1-6, pp. 159-169, 2005. 
[20] B. D. Gupta and A. K. Sharma, "Sensitivity evaluation of a multi-layered surface plasmon resonance-based fiber optic sensor: a theoretical study," Sensors and Actuators B, vol. 107, no. 1, pp. 40-46, 2005.

[21] Rajan, S. Chand, and B. D. Gupta, "Fabrication and characterization of a surface plasmon resonance based fiber-optic sensor for bittering component-Naringin," Sensors and Actuators B, vol. 115, no. 1, pp. 344-348, 2006.

[22] N. Diaz-Herrera, O. Esteban, M. C. Navarrete, M. Le Haitre, and A. Gonzalez-Cano, "In situ salinity measurements in seawater with a fibre-optic probe," Measurement Science and Technology, vol. 17, no. 8, pp. 2227-2232, 2006.

[23] A. K. Sharma, R. Jha, and B. D. Gupta, "Fiber-optic sensors based on surface plasmon resonance: a comprehensive review," IEEE Sensors Journal, vol. 7, pp. 1118-1129, 2007.

[24] E. Kretchmann and H. Reather, "Radiative decay of nonradiative surface plasmons excited by light," Naturforsch, vol. 23, pp. 2135-2136, 1968.

[25] S. A. Zynio, A. V. Samoylov, E. R. Surovtseva, V. M. Mirsky, and Y. M. Shirshov, "Bimetallic layers increase sensitivity of affinity sensors based on surface plasmon resonance," Sensors, vol. 2, no. 2, pp. 62-70, 2002.

[26] A. K. Sharma and B. D. Gupta, "On the performance of different bimetallic combinations in surface plasmon resonance based fiber optic sensors," Journal of Applied Physics, vol. 101, no. 9, Article ID 093111, 6 pages, 2007.

[27] A. K. Sharma, Rajan, and B. D. Gupta, "Influence of dopants on the performance of a fiber optic surface plasmon resonance sensor," Optics Communications, vol. 274, no. 2, pp. 320-326, 2007.

[28] B. Grunwald and G. Holst, "Fibre optic refractive index microsensor based on white-light SPR excitation," Sensors and Actuators A, vol. 113, no. 2, pp. 174-180, 2004.

[29] R. K. Verma, A. K. Sharma, and B. D. Gupta, "Surface plasmon resonance based tapered fiber optic sensor with different taper profiles," Optics Communications, vol. 281, no. 6, pp. 14861491, 2008.

[30] R. K. Verma, A. K. Sharma, and B. D. Gupta, "Modeling of tapered fiber-optic surface plasmon resonance sensor with enhanced sensitivity," IEEE Photonics Technology Letters, vol. 19, no. 22, pp. 1786-1788, 2007.

[31] R. K. Verma and B. D. Gupta, "Theoretical modeling of a bidimensional U-shaped surface plasmon resonance based fibre optic sensor for sensitivity enhancement," Journal of Physics D, vol. 41, Article ID 095106, 2008.

[32] J. Homola and R. Slavik, "Fibre-optic sensor based on surface plasmon resonance," Electronics Letters, vol. 32, no. 5, pp. 480482, 1996.

[33] H.-Y. Lin, W.-H. Tsai, Y.-C. Tsao, and B.-C. Sheu, "Sidepolished multimode fiber biosensor based on surface plasmon resonance with halogen light," Applied Optics, vol. 46, no. 5, pp. 800-806, 2007.

[34] Y.-C. Lin, Y.-C. Tsao, W.-H. Tsai, T.-S. Hung, K.-S. Chen, and S.-C. Liao, "The enhancement method of optical fiber biosensor based on surface plasmon resonance with cold plasma modification," Sensors and Actuators B, vol. 133, no. 2, pp. 370-373, 2008.

[35] M.-H. Chiu, C.-H. Shih, and M.-H. Chi, "Optimum sensitivity of single-mode D-type optical fiber sensor in the intensity measurement," Sensors and Actuators B, vol. 123, no. 2, pp. 1120-1124, 2007.
[36] M.-H. Chiu and C.-H. Shih, "Searching for optimal sensitivity of single-mode D-type optical fiber sensor in the phase measurement," Sensors and Actuators B, vol. 131, no. 2, pp. 596-601, 2008.

[37] D. J. Gentleman and K. S. Booksh, "Determining salinity using a multimode fiber optic surface plasmon resonance dipprobe," Talanta, vol. 68, no. 3, pp. 504-515, 2006.

[38] H. Suzuki, M. Sugimoto, Y. Matsui, and J. Kondoh, "Effects of gold film thickness on spectrum profile and sensitivity of a multimode-optical-fiber SPR sensor," Sensors and Actuators B, vol. 132, no. 1, pp. 26-33, 2008.

[39] K. Kurihara, H. Ohkawa, Y. Iwasaki, O. Niwa, T. Tobita, and K. Suzuki, "Fiber-optic conical microsensors for surface plasmon resonance using chemically etched single-mode fiber," Analytica Chimica Acta, vol. 523, no. 2, pp. 165-170, 2004.

[40] T. Abrahamyan and Kh. Nerkararyan, "Surface plasmon resonance on vicinity of gold-coated fiber tip," Physics Letters A, vol. 364, no. 6, pp. 494-496, 2007.

[41] A. Hassani and M. Skorobogatiy, "Design criteria for microstructured-optical-fiber-based surface-plasmonresonance sensors," Journal of the Optical Society of America B, vol. 24, no. 6, pp. 1423-1429, 2007.

[42] B. Gauvreau, A. Hassani, M. F. Fehri, A. Kabashin, and M. Skorobogatiy, "Photonic bandgap fiber-based surface plasmon resonance sensors," Optics Express, vol. 15, no. 18, pp. 1141311426, 2007.

[43] Y. S. Dwivedi, A. K. Sharma, and B. D. Gupta, "Influence of skew rays on the sensitivity and signal-to-noise ratio of a fiberoptic surface-plasmon-resonance sensor: a theoretical study," Applied Optics, vol. 46, no. 21, pp. 4563-4569, 2007.

[44] S. K. Ozdemir and G. Turhan-Sayan, "Temperature effects on surface plasmon resonance: design considerations for an optical temperature sensor," Journal of Lightwave Technology, vol. 21, no. 3, pp. 805-814, 2003.

[45] A. K. Sharma and B. D. Gupta, "Theoretical model of a fiber optic remote sensor based on surface plasmon resonance for temperature detection," Optical Fiber Technology, vol. 12, no. 1, pp. 87-100, 2006.

[46] Rajan, S. Chand, and B. D. Gupta, "Surface plasmon resonance based fiber-optic sensor for the detection of pesticide," Sensors and Actuators B, vol. 123, no. 2, pp. 661-666, 2007.

[47] T. Matsushita, T. Nishikawa, H. Yamashita, J. Kishimoto, and Y. Okuno, "Development of new single-mode waveguide surface plasmon resonance sensor using a polymer imprint process for high-throughput fabrication and improved design flexibility," Sensors and Actuators B, vol. 129, no. 2, pp. 881887, 2008.

[48] Y.-J. He, Y.-L. Lo, and J.-F. Huang, "Optical-fiber surfaceplasmon-resonance sensor employing long-period fiber gratings in multiplexing," Journal of the Optical Society of America $B$, vol. 23, no. 5, pp. 801-811, 2006.

[49] B. Spackova, M. Piliarik, P. Kvasnicka, C. Themistos, M. Rajarajan, and J. Homola, "Novel concept of multi-channel fiber optic surface plasmon resonance sensor," Sensors and Actuators B, vol. 139, no. 1, pp. 199-203, 2009. 

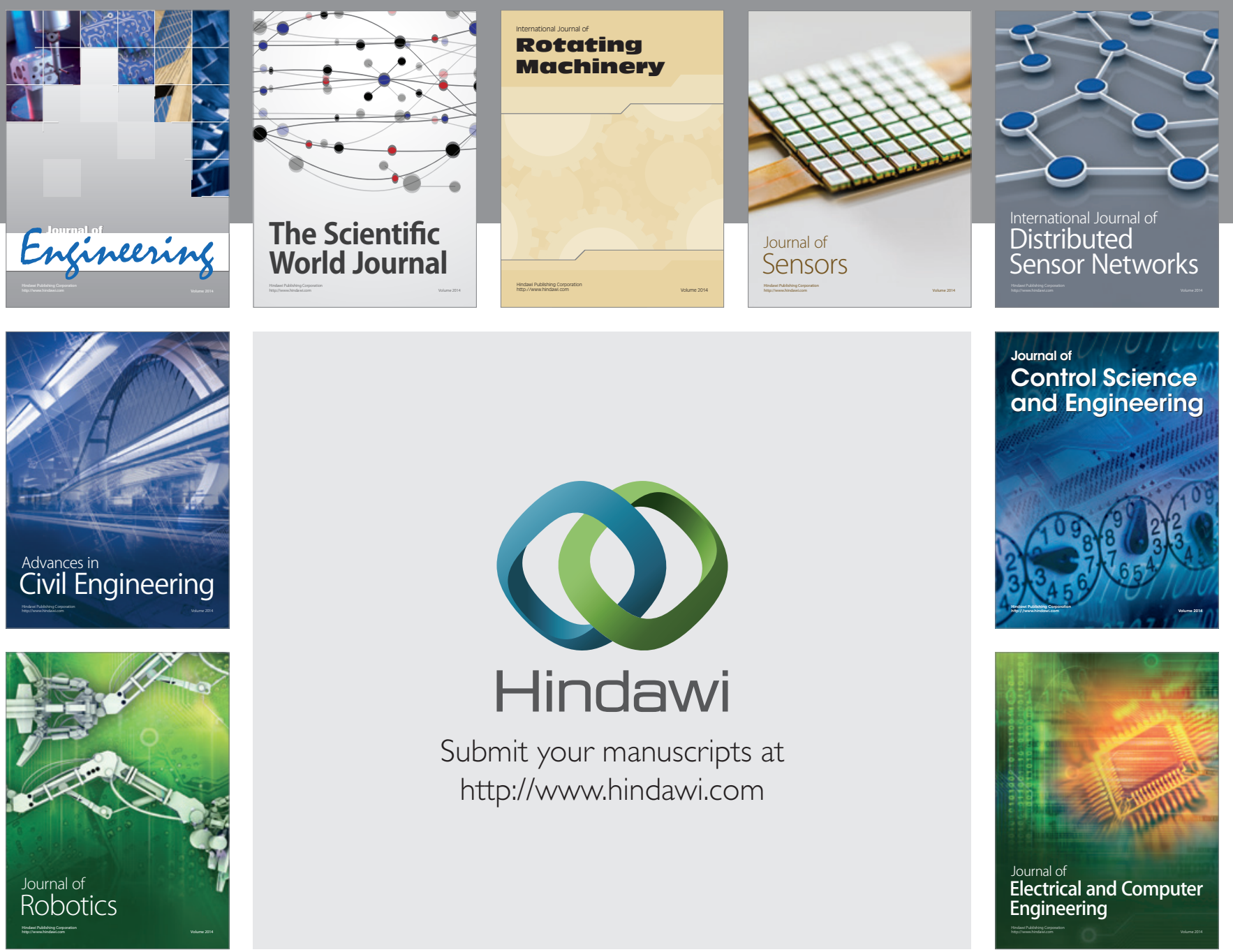

Submit your manuscripts at

http://www.hindawi.com
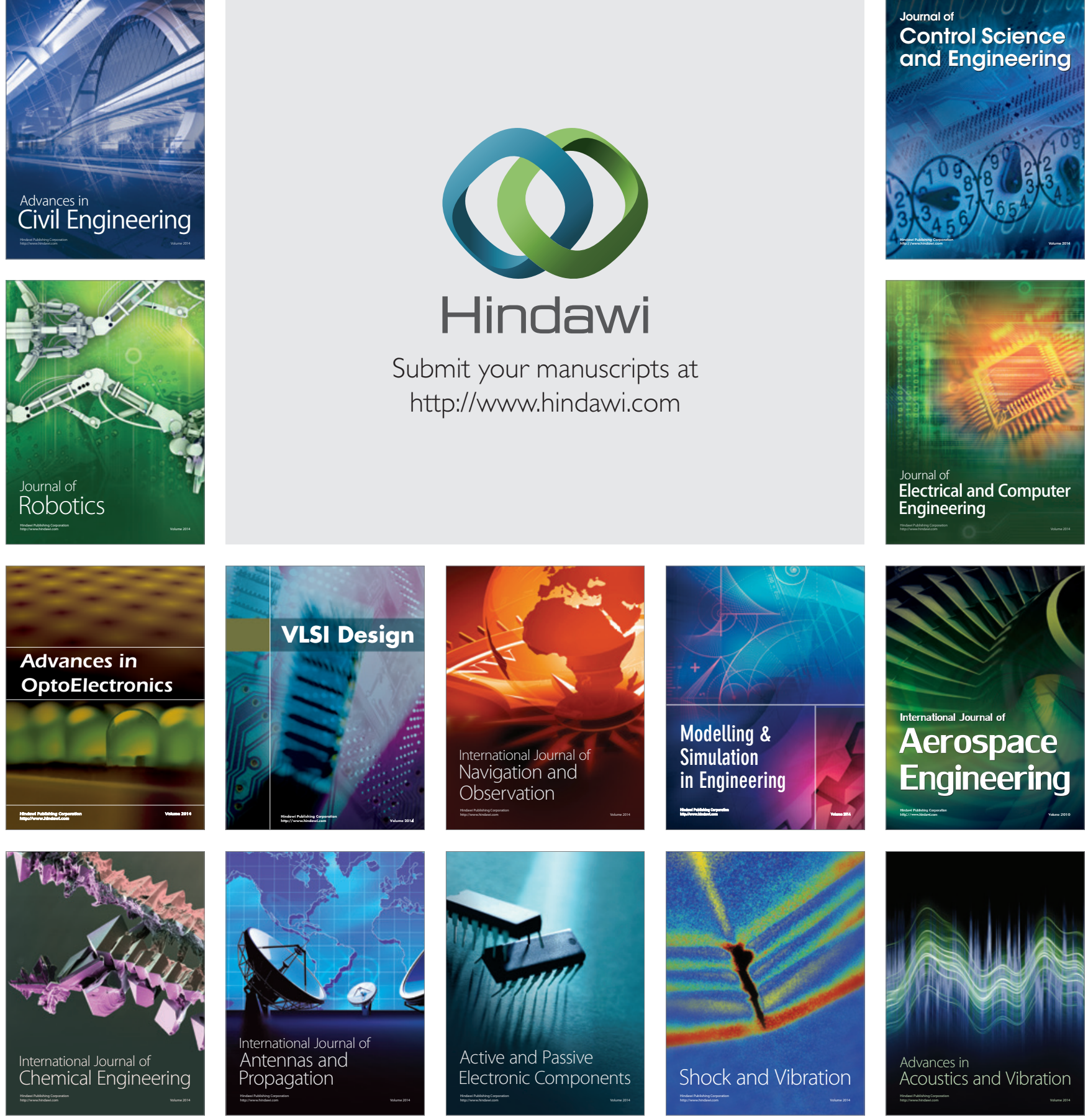